\title{
Investigation into the acute effects of total and partial energy restriction on postprandial metabolism among overweight/obese participants
}

\author{
Rona Antoni ${ }^{1}$, Kelly L. Johnston ${ }^{2}$, Adam L. Collins ${ }^{1}$ and M. Denise Robertson ${ }^{1 *}$ \\ ${ }^{1}$ Nutrition, Metabolism and Diabetes Research Group, Faculty of Health and Medical Sciences, University of Surrey, \\ Guildford GU2 7WG, UK \\ ${ }^{2}$ Lighterlife UK Ltd, Cavendish House, Parkway, Harlow Business Park, Essex CM19 5QF, UK \\ (Submitted 14 October 2015 - Final revision received 2 December 2015 - Accepted 7 December 2015 - First published online 28 January 2016)
}

\section{Abstract}

The intermittent energy restriction (IER) approach to weight loss involves short periods of substantial (75-100\%) energy restriction (ER) interspersed with normal eating. This study aimed to characterise the early metabolic response to these varying degrees of ER, which occurs acutely and prior to weight loss. Ten (three female) healthy, overweight/obese participants (36 (SEM 5) years; $29 \cdot 0$ (SEM $1 \cdot 1$ ) kg/ $\mathrm{m}^{2}$ ) took part in this acute three-way crossover study. Participants completed three 1-d dietary interventions in a randomised order with a 1-week washout period: isoenergetic intake, partial $75 \%$ ER and total $100 \%$ ER. Fasting and postprandial (6-h) metabolic responses to a liquid test meal were assessed the following morning via serial blood sampling and indirect calorimetry. Food intake was also recorded for two subsequent days of ad libitum intake. Relative to the isoenergetic control, postprandial glucose responses were increased following total ER $(+142 \% ; P=0.015)$ and to a lesser extent after partial ER $(+76 \%$; $P=0.051)$. There was also a delay in the glucose time to peak after total ER only $(P=0.024)$. Both total and partial ER interventions produced comparable reductions in postprandial TAG responses $(-75$ and $-59 \%$, respectively; both $P<0.05)$ and 3-d energy intake deficits of approximately $30 \%$ (both $P=0 \cdot 015$ ). Resting and meal-induced thermogenesis were not significantly affected by either ER intervention. In conclusion, our data demonstrate the ability of substantial ER to acutely alter postprandial glucose-lipid metabolism (with partial ER producing the more favourable overall response), as well as incomplete energy-intake compensation amongst overweight/obese participants. Further investigations are required to establish how metabolism adapts over time to the repeated perturbations experienced during IER, as well as the implications for long-term health.

\section{Key words: Intermittent energy restriction: Intermittent fasting: Alternate-day fasting: Type 2 diabetes: CVD: Cardiometabolic risk}

In recent years, intermittent energy restriction (IER) has become the subject of considerable research interest as an alternative to the more conventional continuous energy restriction (ER) approaches to weight loss ${ }^{(1)}$. An array of IER protocols have been studied; each protocol involves intermittent periods of very low (or no) energy intake followed by normal eating, most commonly for $1^{(2)}$ or $2 \mathrm{~d} /$ week $^{(3,4)}$, or on alternate days ${ }^{(5,6)}$. Consequently, dieters undergo repeated ER-re-feed cycles over the course of the week. However, little is known about the effects of this altered eating pattern on postprandial glucose-lipid metabolism, which is pertinent, given the growing evidence base implicating both as independent discriminators of CVD risk $^{(7,8)}$.

Additionally, studies of very low-energy diets demonstrate dramatic changes in metabolic control within overweight/obese cohorts, long before significant weight loss occurs ${ }^{(\mathcal{Q}, 10)}$. This acute timecourse suggests a primary role of dietary ER, or more specifically the creation of a profound negative energy balance, as a primary mediator underlying these early effects. Understanding the timecourse over which such metabolic changes occur during ER is important for our understanding of the metabolic adaptation which occurs during weight loss. It is well established that short-term fasting elicits a coordinated metabolic response to the accompanying energy deficit, resulting in increased fatty acid mobilisation from adipose tissue, as well as a shift in fuel utilisation towards fatty acid oxidation (FAO) and ketogenesis (reviewed by Soeters et $\left.a l^{(11)}\right)$. There is also a marked decline in peripheral insulin sensitivity and glucose tolerance ${ }^{(12-16)}$. From an evolutionary standpoint, these reciprocal adaptations in glucose-lipid metabolism serve to conserve glucose, and thus limit the utilisation of protein stores. They are reversed through food re-introduction, but this may require upwards of $48 \mathrm{~h}$ of re-feeding ${ }^{(12)}$.

The majority of acute research to date has either involved healthy-weight individuals or more prolonged ( $\geq 48 \mathrm{~h}$ ) total fasting intervals, as this is the timecourse in which the short-term adaptations to fasting occur and become maximal ${ }^{(11)}$. IER has predominantly been investigated as a weight-loss strategy within overweight/obese cohorts. The majority of dietary protocols used in these studies allow a small amount of food intake in an attempt to improve the tolerability and compliance to IER, such that energy is substantially (but not completely) restricted. This has the

Abbreviations: 3 -OHB, 3- $\beta$-hydroxybutyrate; dAUC, decremental AUC; ER, energy restriction; iAUC, incremental AUC; IER, intermittent energy restriction; REE, resting energy expenditure.

*Corresponding author: M. D. Robertson, email m.robertson@surrey.ac.uk 
potential to alter the response which has been well defined for more prolonged fasting intervals, and thus the subsequent postprandial response during re-feeding. Acutely, this is best assessed using a within-subject study design, which to our knowledge has not been performed.

With this in mind the present cross-over study, conducted in ten overweight or obese participants, aimed to characterise the early metabolic response to varying degrees of ER by assessing postprandial responses to a liquid mixed test meal after $1 \mathrm{~d}$ of total (100\%) and partial (75\%) ER. Secondary outcomes included subsequent energy intake compensation and thermogenic responses, potential limiting factors for the long-term weight-loss efficacy of IER.

\section{Methods}

\section{Participants}

In total, fourteen (six female) healthy, overweight or obese participants aged 18-60 years were initially recruited to the study from the University of Surrey and wider community. Four (three female) participants did not complete the study because of non-compliance ( $n$ 1) or cannulation difficulties ( $n$ 3), culminating in ten study completers (see Table 1 for participant

Table 1. Participant characteristics taken at the pre-trial visit (Mean values with their standard errors)

\begin{tabular}{|c|c|c|c|c|c|c|}
\hline & \multicolumn{2}{|c|}{ All participants $(n 10)$} & \multicolumn{2}{|c|}{ Males $(n 7)$} & \multicolumn{2}{|c|}{ Females $(n 3)$} \\
\hline & Mean & SEM & Mean & SEM & Mean & SEM \\
\hline Age (years) & 36 & $4 \cdot 6$ & 42 & $5 \cdot 2$ & 24 & $2 \cdot 0$ \\
\hline Weight (kg) & $91 \cdot 2$ & $5 \cdot 6$ & $97 \cdot 4$ & 6.5 & 76.6 & 4.4 \\
\hline BMI $\left(\mathrm{kg} / \mathrm{m}^{2}\right)$ & 29.1 & 0.8 & 29.0 & $1 \cdot 1$ & 29.5 & 0.6 \\
\hline Body fat $(\%)^{*}$ & 29.8 & $2 \cdot 3$ & $26 \cdot 3$ & 1.9 & 37.9 & $2 \cdot 1$ \\
\hline
\end{tabular}

* Measured using bioimpedance (Tanita MC180A; Tanita Corp). characteristics). Participants were weight stable ( $\pm 2 \mathrm{~kg}$ ) over the preceding 3 months and had no significant medical history. Health status was determined by medical questionnaire and screening blood sample. Restrained eaters were identified and excluded using the Dutch Eating Behaviour Questionnaire (cut-off: >4) as potential confounders for the secondary outcome $^{(17)}$. To control for the potential influence of the menstrual cycle between visits, recruited female participants were either post-menopausal or taking oral contraceptives. The study was approved by the University of Surrey Ethics Committee and conducted in accordance with the guidelines laid down in the Declaration of Helsinki. Written informed consent was obtained from all participants.

\section{General protocol}

The study was a three-way, randomised, cross-over study (Fig. 1), in which the participants completed three 1-d dietary interventions in a random order with a minimum 1-week washout: $1 \mathrm{~d}$ of isoenergetic intake (0\% ER), which served as the control; $1 \mathrm{~d}$ of total (100\%) ER; and $1 \mathrm{~d}$ of partial (75\%) ER. Metabolic assessments were conducted on the following day (Day 2). To assess for short-term energy compensation, dietary intakes were recorded over a 3-d period, which encompassed each controlled intake day (Day 1), while participants were at the research unit (Day 2) and upon resumption of ad libitum intake (Days 2 and 3). To ensure familiarity with study procedures, participants first completed a pre-study test run, which was identical in design to the isoenergetic intervention.

Day 1: experimental diets. Energy requirements were calculated using the Henry predictive equation for $\mathrm{BMR}^{(18)}$ as this forms a part of the updated estimated average requirement for energy ${ }^{(19)}$. Participants were asked to minimise their activity levels during each intervention period, and so the physical

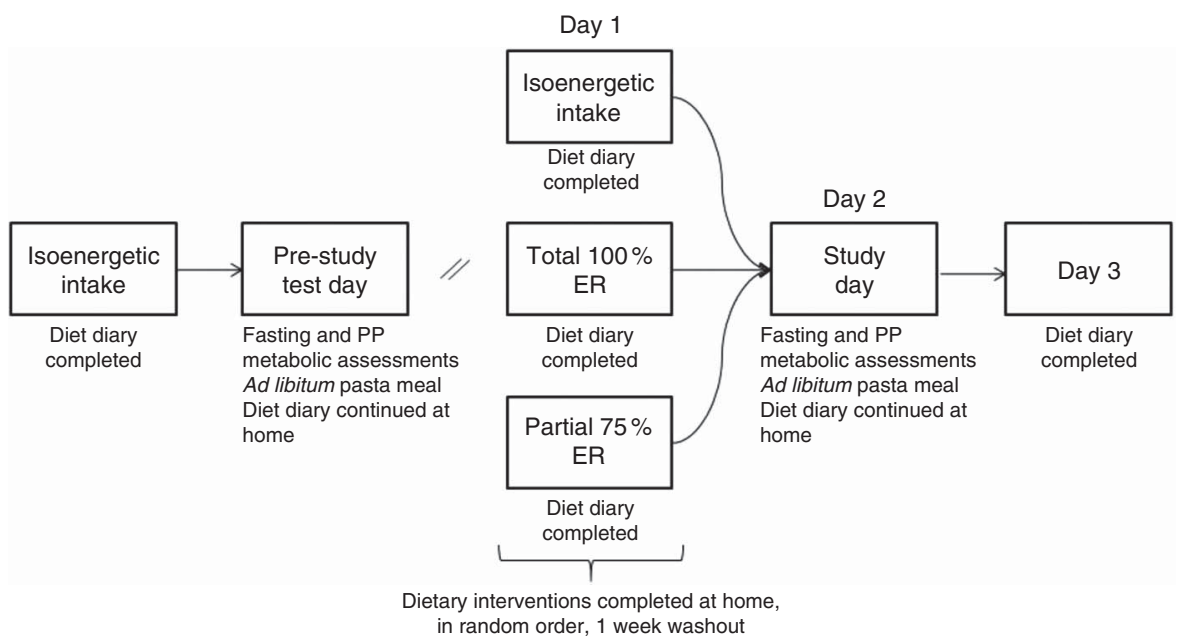

Fig. 1. Schematic overview of study. A randomised, cross-over study where participants completed three dietary interventions in a random order: $1 \mathrm{~d}$ of isoenergetic intake ( $0 \%$ energy restriction (ER)), which served as the control; $1 \mathrm{~d}$ of total $(100 \%)$ ER; and $1 \mathrm{~d}$ of partial $(75 \%)$ ER. Metabolic assessments were conducted on the following day (Day 2). To assess for short-term energy compensation, dietary intakes were recorded over a 3-d period, which encompassed each controlled intake day (Day 1), while participants were at the research unit (Day 2) and upon resumption of ad libitum intake (Days 2 and 3). There was a minimum 1-week washout period. To ensure familiarity with study procedures, participants first completed a pre-study test run, which was identical in design to the isoenergetic intervention. PP, postprandial. 
activity levels used to calculate daily energy requirements were representative of sedentary activity levels ${ }^{(19)}$.

Isoenergetic control diet (O\% ER): each participant was supplied with an isoenergetic diet comprised of commonly consumed food and drinks (11040 (SEM 1482) kJ, 327 (SEM 12) g carbohydrate (55\% of total energy), 99 (SEM 3) g protein (15\% of total energy) and 97 (SEM 5) g fat (30\% of total energy)) providing $100 \%$ of their estimated isoenergetic needs.

Total (100\%) ER: participants started their fast from 20.00 hours the night before their dietary intervention day until 08.00 hours on the morning of their study day, totalling $36 \mathrm{~h}$.

Partial (75\%) ER: participants consumed four commercially available LighterLife ${ }^{\mathrm{TM}}$ FoodPacks $(2638 \mathrm{~kJ}, 58 \mathrm{~g}$ carbohydrate (37\% of total energy), $54 \mathrm{~g}$ protein ( $35 \%$ of total energy), $17 \mathrm{~g}$ fat ( $28 \%$ of total energy)), which provided approximately $25 \%$ of their estimated isoenergetic needs. The degree of ER chosen is comparable with that used by previously published IER weightloss trials ${ }^{(3,4,6)}$.

During each dietary intervention, participants were advised to consume sufficient amounts of non-caloric fluids and to abstain from alcohol. During the isoenergetic and partial ER interventions, participants ate their last meal no later than 20.00 hours.

Day 2: metabolic studies. Participants attended the Surrey Clinical Research Centre on the morning (08.00 hours) after each controlled energy intake day. Body weight and composition were measured by bioimpedance (Tanita MC180A; Tanita Corp.). Resting measurements of energy expenditure and substrate utilisation were then measured via indirect calorimetry (GEM Nutrition) after participants had rested for $30 \mathrm{~min}$. An indwelling cannula was then inserted following which the first (fasting) sample was collected. A liquid, mixed test meal was provided ( $400 \mathrm{ml}$ Fortisip; Nutricia: $2510 \mathrm{~kJ}, 74 \mathrm{~g}$ carbohydrate, $24 \mathrm{~g}$ protein, $23 \mathrm{~g}$ fat). Serial measurements of energy expenditure, substrate utilisation and blood samples were obtained at regular intervals from the start of the test meal over the next $360 \mathrm{~min}$. Participants were then presented with a large pre-weighed ad libitum homogeneous pasta meal in excess of normal portions (whole dish provided: $9950 \mathrm{~kJ}, 334 \mathrm{~g}$ carbohydrate, $83 \mathrm{~g}$ protein, $74 \mathrm{~g}$ fat) and instructed to eat until comfortably full. They were also instructed to resume habitual intake after leaving the unit and to keep a diet diary until midnight of the next day (Day 3).

\section{Experimental techniques and calculations}

Indirect calorimetry. Energy expenditure and substrate utilisation were measured using the Europa Gaseous Exchange Monitor (GEM Nutrition), an open-circuit indirect calorimeter based on the ventilated flow-through technique ${ }^{(20)}$. For the duration of the study day, participants were asked to minimise activity and to be rested before all measurements. Resting (fasted) measures were taken over $20 \mathrm{~min}$ and in accordance with methodological recommendations by Compher et al. ${ }^{(21)}$. Postprandial gaseous exchange measurements were taken over $10 \mathrm{~min}$ every $30 \mathrm{~min}$ for the first $180 \mathrm{~min}$ after the test meal and then hourly thereafter until the end of the 360-min postprandial period. Resting and meal-induced thermogenesis (MIT) were calculated using the modified Weir equation ${ }^{(22)}$. Fasting and postprandial substrate oxidation were calculated using the non-protein stoichiometric equations from Frayn ${ }^{(23)}$. These equations assume negligible contributions from gluconeogenesis, protein oxidation and ketogenesis; whilst these assumptions may not hold true following substantial ER, evidence from studies over longer (72-h) fasting durations suggest that the error introduced by not accounting for the latter two metabolic processes would be minimal ${ }^{(14)}$.

Blood biochemistry and calculations. Serial blood measurements were taken at baseline and then at regular intervals of 15 , 30, 60, 90, 120, 180, 240, 300 and $360 \mathrm{~min}$. Blood samples were collected into potassium EDTA tubes (for the analysis of TAG, NEFA, insulin and 3- $\beta$-hydroxybutyrate (3-OHB)) and sodium oxalate tubes (for glucose analysis). Samples were centrifuged for $15 \mathrm{~min}$ at $2500 \mathrm{rpm}$ and separated; plasma aliquots were then stored at $-20^{\circ} \mathrm{C}$ with a subset intended for 3-OHB analysis stored at $-80^{\circ} \mathrm{C}$. Samples were batch analysed upon study completion with all samples from an individual participant included in the same assay. Metabolites were analysed using the following methods: insulin using RIA (Millipore; intra/ inter-assay CV 6 and 8\%); glucose, TAG and NEFA using commercially available kits (Instrumentation Laboratory) for the ILAB650 (Instrumentation Laboratory; intra/inter-assay CV all $<4$ and $<7 \%$ ); and 3-OHB using commercially available kits (Randox) for the Cobas MIRA (Roche; intra/inter-assay CV 6 and 9\%). Incremental AUC (iAUC) was calculated to quantify postprandial metabolic responses ${ }^{(24)}$.

Dietary analyses. All diet diary analyses were carried out in Diet Plan 6 (Forestfield Software) using the McCance and Widdowson's composition of foods integrated data set. Participants recorded intake in validated diet diaries which included pictorial guides to aid portion size estimations when exact weights could not be provided. Nutritional intake information recorded in diet diaries (Days 1-3), from the weighted ad libitum meal intake (Day 2) and test meal (Day 2) were aggregated. This information was then used to calculate daily and cumulative 3-d energy consumption, expressed as the percentage of estimated isoenergetic needs derived from the Henry equation ${ }^{(18)}$.

Statistical analyses. Statistical analyses were carried out using IBM SPSS version 22 (SPSS Inc.). In view of the small sample size, non-parametric testing was deemed the most appropriate for the analysis of summary measures. Comparisons between the three experimental arms were conducted using the Friedman test with the Wilcoxon signed-ranks test used for subsequent pairwise comparisons. A Bonferroni correction was applied to correct for multiple post hoc testing. For timecourse data, where no robust non-parametric test is able to examine main and interaction effects, repeated-measures ANOVA was used with a Sidak correction applied to post hoc pairwise comparisons. Before this, any non-normally distributed data were $\log$ transformed. Diet and time point (for timecourse data) were used as within-subject factors. Statistical significance was accepted at the 5\% level. All results are presented as mean values with their standard errors. 


\section{Sample size}

Based on the change in the postprandial TAG iAUC between isoenergetic and partial (75\%) ER conditions: ten participants in a cross-over design study, $\alpha=0.05$, two-sided, would give a $90 \%$ power of detecting a difference in response of 47 units assuming a standard deviation of the response to be 41 units. In retrospect, the actual mean change in TAG was 89 (SEM 13) units, resulting in a study power $>99 \%$ for this primary outcome variable.

\section{Results}

\section{Dietary intakes}

Table 2 displays day-by-day and cumulative energy consumption across each 3-d study period. Reported dietary intakes during controlled energy intake days (Day 1) were close to prescribed amounts. On Day 2, participants over-consumed by 22.7 (SEM 7.5) \% above estimated daily energy requirements after their total ER (fast) day $(P=0.010 v$. iso) and by +10.0 (SEM 5.7$) \%$ following their partial ER day (non-significant $v$. iso). On Day 3, (Mean values with their standard errors; $n$ 10) no significant differences in energy intake were detected across the three dietary interventions when expressed either in $\mathrm{kJ}$ or relative to isoenergetic requirements, with participants seemingly under-consuming after all three interventions. Overall, cumulative 3-d intakes were significantly lower following both total and partial ER interventions relative to the isoenergetic control leg, with participants remaining in comparable net energy deficits of $-28 \cdot 4$ (SEM 5.2) \% and $-30 \cdot 3$ (SEM 3.1)\%, respectively (both $P=0.015 v$. iso). No significant differences in any dietary intake measure were noted between total and partial ER interventions.

\section{Fasting metabolism}

Fasting substrate levels and fuel oxidation, assessed on the morning after each intervention (Day 2), are presented in Table 3. Relative to the isoenergetic control, total ER led to a significant reduction in levels of fasting plasma glucose $(P=0.022 v$. iso $)$ and TAG $(P=0.039 v$. iso), whereas NEFA levels and 3-OHB were higher $(P=0.022$ and 0.015 , respectively, $v$. iso). Accordingly, fasting RQ was significantly lower following total ER $v$. iso only $(P=0.024)$, indicative of higher rates of fat oxidation. Following

Table 2. Daily and cumulative 3-d energy intakes during isoenergetic, total energy restriction (ER) and partial ER dietary interventions. Broken down into individual and cumulative energy consumption during the controlled energy intake day, 1 and $2 \mathrm{~d}$ later

\begin{tabular}{|c|c|c|c|c|c|c|}
\hline & \multicolumn{2}{|c|}{ Isoenergetic } & \multicolumn{2}{|c|}{ Partial (75\%) ER } & \multicolumn{2}{|c|}{ Total (100\%) ER } \\
\hline & Mean & SEM & Mean & SEM & Mean & SEM \\
\hline \multicolumn{7}{|l|}{ Controlled intake (Day 1 ) } \\
\hline Energy intake $(\mathrm{kJ} / \mathrm{d})$ & 11040 & 468 & $2688^{a, b}$ & 43 & $118^{\mathrm{a}, \mathrm{c}}$ & 73 \\
\hline$\%$ ER achieved ${ }^{*}$ & +0.4 & 0.3 & $-75 \cdot 2^{a, b}$ & 1.0 & $-98 \cdot 8^{\mathrm{a}, \mathrm{c}}$ & 0.7 \\
\hline \multicolumn{7}{|l|}{1 day post (Day 2) } \\
\hline Ad libitum meal (kJ) & 4929 & 354 & 5341 & 342 & $5946^{a}$ & 534 \\
\hline Total $24 \mathrm{~h}$ intake $(\mathrm{kJ} / \mathrm{d})$ & 11816 & 1057 & 12154 & 905 & 13522 & 1036 \\
\hline$\% 24 \mathrm{~h}$ energy balance* & +7.5 & 8.6 & $+10 \cdot 0$ & $5 \cdot 7$ & $+22 \cdot 7$ & 7.5 \\
\hline \multicolumn{7}{|l|}{2 days post (Day 3 ) } \\
\hline Total $24 \mathrm{~h}$ intake $(\mathrm{kJ} / \mathrm{d})$ & 9675 & 1089 & 8428 & 792 & 9914 & 1026 \\
\hline$\% 24$ h energy balance* & $-12 \cdot 6$ & $8 \cdot 6$ & $-23 \cdot 1$ & $6 \cdot 8$ & -8.9 & 9.0 \\
\hline \multicolumn{7}{|l|}{ Cumulative $3 \mathrm{~d}$ total } \\
\hline Total daily intake $(\mathrm{kJ} / \mathrm{d})$ & 32532 & 2257 & $23026^{a}$ & 1404 & $23556^{a}$ & 1941 \\
\hline Net $3 d$ energy balance ${ }^{*}$ & -1.5 & $5 \cdot 1$ & $-30 \cdot 3^{a}$ & $3 \cdot 1$ & $-28.4^{\mathrm{a}}$ & $5 \cdot 2$ \\
\hline
\end{tabular}

a,b,c Significantly different to ${ }^{\mathrm{a}}$ isoenergetic $(P<0.05),{ }^{\mathrm{b}}$ total $(100 \%)$ ER $(P<0.05),{ }^{\mathrm{c}}$ partial $(75 \%)$ ER $(P<0.05)$.

* Expressed as the percentage relative to participants' estimated daily requirement for weight maintenance. Comparisons made using the Friedman test, with post hoc Wilcoxon signed-ranks testing used for subsequent pairwise comparisons (Bonferroni correction applied).

Table 3. Fasting substrate levels and fuel oxidation the morning after 1 day of isoenergetic intake, total energy restriction (ER) and partial ER (i.e Day 2) (Mean values with their standard errors)

\begin{tabular}{|c|c|c|c|c|c|c|}
\hline & \multicolumn{2}{|c|}{ Isoenergetic } & \multicolumn{2}{|c|}{ Partial (75\%) ER } & \multicolumn{2}{|c|}{ Total (100\%) ER } \\
\hline & Mean & SEM & Mean & SEM & Mean & SEM \\
\hline Glucose (mmol/l) & $4 \cdot 7$ & 0.1 & $4 \cdot 4^{\mathrm{a}}$ & 0.1 & $4 \cdot 3^{\mathrm{a}}$ & 0.2 \\
\hline Insulin (pmol/l) & 89.7 & 9.2 & $69 \cdot 1$ & 8.0 & 74.0 & 12.4 \\
\hline TAG $(\mathrm{mmol} / \mathrm{l})$ & 1.5 & 0.2 & $1 \cdot 2^{\mathrm{a}}$ & 0.2 & $1 \cdot 2^{\mathrm{a}}$ & 0.3 \\
\hline NEFA (mmol//l) & 0.63 & 0.07 & $0.73^{\mathrm{b}}$ & 0.06 & $1.01^{\mathrm{a}, \mathrm{c}}$ & 0.11 \\
\hline 3-OHB (mmol/l) & 0.05 & 0.02 & $0 \cdot 26^{a, b}$ & 0.07 & $0.56^{\mathrm{a}, \mathrm{c}}$ & 0.11 \\
\hline $\mathrm{NP} R Q\left(\mathrm{VO}_{2} / \mathrm{VCO}_{2}\right)^{*}$ & 0.89 & 0.03 & 0.84 & 0.02 & $0.81^{\mathrm{a}}$ & 0.03 \\
\hline
\end{tabular}

3-OHB, 3- $\beta$-hydroxybutyrate; NP RQ, non-protein respiratory quotient.

a,b,c Significantly different to ${ }^{\mathrm{a}}$ isoenergetic $(P<0.05),{ }^{\mathrm{b}}$ total $(100 \%)$ ER $(P<0.05),{ }^{\mathrm{c}}$ partial $(75 \%)$ ER $(P<0.05)$.

* NP RQ: $0 \cdot 7=$ pure fat oxidation; $1 \cdot 0=$ pure carbohydrate oxidation. Comparisons made using the Friedman test, with post hoc Wilcoxon signed-ranks testing used for subsequent pairwise comparisons (Bonferroni correction applied). $n$ 10, plasma concentrations; $n 8, \mathrm{NP} R Q$. 
partial ER, similar trends were noted but the rise in plasma NEFA did not reach statistical significance $v$. iso. Plasma NEFA and 3-OHB were significantly higher after total ER when compared with partial ER (both $P=0.015$ ); no other statistical differences between the ER interventions were noted.

\section{Postprandial substrate metabolism}

Postprandial substrate and insulinaemic responses to the liquid test meal, given the morning after each ER intervention, are presented in Fig. 2(a-d) along with main and interaction effects for timecourse data.

\section{Glucose and insulin responses}

There were main effects of intervention on postprandial glucose iAUC $(P=0.001$; Fig. 2(a)); glucose iAUC in response to the liquid test meal was significantly greater following total ER relative to iso (402 (SEM 64) v. 166 (SEM 35) $\mathrm{mmol} / \mathrm{min}$ per litre; $P=0.015)$ and tended to be greater than the partial ER leg (294 (SEM 40$) \mathrm{mmol} / \mathrm{min}$ per litre; $P=0 \cdot 051$ ). A non-significant trend in favour of greater glucose iAUC following partial ER $v$. iso was also found $(P=0.051)$. Postprandial glucose time to peak was significantly delayed following total ER relative to both iso $(P=0.024)$ and partial $\mathrm{ER}(P=0.018)$ interventions. No significant differences in plasma insulin iAUC were detected across the dietary interventions (Fig. 2(b)).

\section{TAG responses}

There were highly significant main effects of intervention on TAG iAUC ( $P=0 \cdot 001$; Fig. 2(c)); compared with iso (131 (SEM 20) mmol/ min per litre), postprandial iAUC was significantly lower following both total (32 (SEM 11) $\mathrm{mmol} / \mathrm{min}$ per litre, $P=0.015 v$. iso) and partial $(54 \pm 19 \mathrm{mmol} / \mathrm{min}$ per litre, $P=0.039 v$. iso) ER interventions. TAG iAUC did not differ between total and partial ER.

\section{NEFA}

There were significant main effects of intervention on NEFA postprandial decremental AUC (dAUC) $(P=0.002$; Fig. 2(d)); following total ER, plasma NEFA dAUC was significantly different compared with iso (-195 (SEM 36) v. -90 (SEM 22) $\mathrm{mmol} / \mathrm{min}$ per litre; $P=0.037$ ) and partial ER ( -101 (SEM 18) $\mathrm{mmol} / \mathrm{min}$ per litre; $P=0 \cdot 015)$. dAUC did not differ significantly between partial ER and iso interventions.

\section{Postprandial substrate oxidation and energy metabolism}

Postprandial substrate oxidation responses ( $n$ 8) following the liquid test meal are presented in Fig. 3(a-c). Indirect calorimetry data for two participants were excluded after data loss due to equipment failure. No significant main effects were observed for postprandial fat oxidation (Fig. 3(a)). A significant main effect of intervention was observed for postprandial carbohydrate oxidation ( $P=0.011$; Fig. 3(c)), which was lower following total ER $v$. iso only $(P=0 \cdot 036)$. Postprandial plasma 3-OHB concentrations (Fig. 3(b)) paralleled NEFA responses (Fig. 2(d)) with circulating levels suppressed to the same extent across the dietary interventions by approximately $180 \mathrm{~min}$, before rising markedly thereafter following total and partial ER interventions only. Statistical analyses showed significant main effects of intervention $(P<0.001)$ on postprandial 3-OHB dAUC; following total ER, plasma 3-OHB dAUC was significantly different compared with iso (-133 (SEM 36) (a)

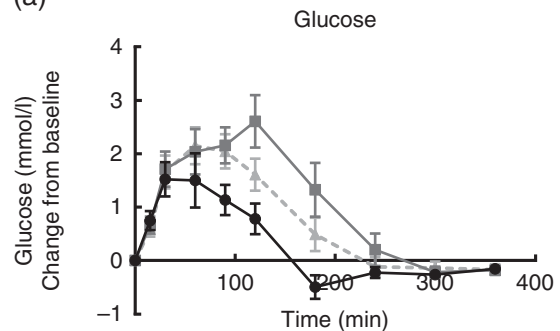

(c)

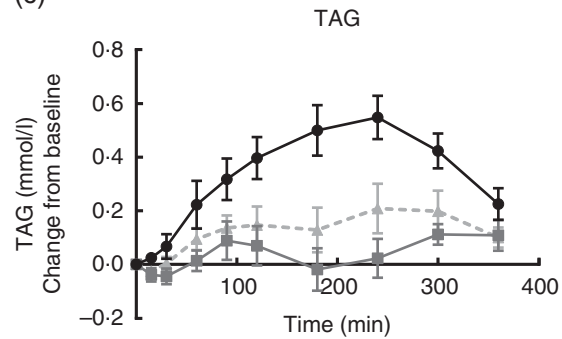

(b)

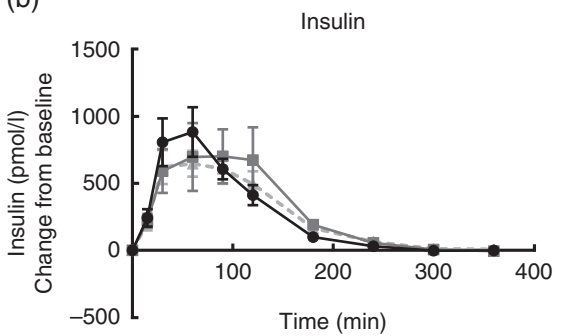

(d)

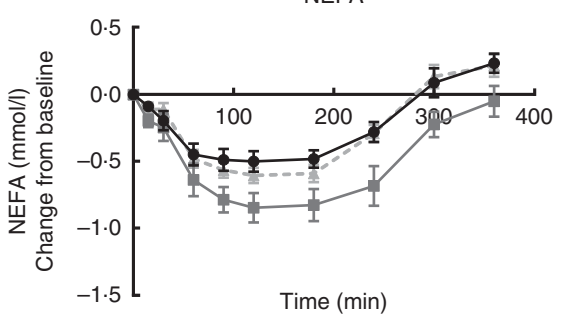

Fig. 2. Postprandial (6-h) substrate responses to liquid test meal $(2510 \mathrm{~kJ}$ ( $600 \mathrm{kcal}), 74 \mathrm{~g}$ carbohydrate, $24 \mathrm{~g}$ protein, $23 \mathrm{~g}$ fat) following $1 \mathrm{~d}$ of total $100 \%$ energy restriction (ER) (- - ) and partial 75\% ER (- $\left.A_{-}^{-}\right) v$. an isoenergetic (iso) control diet $(--)$ (a) Plasma glucose: there was a significant diet $\times$ time interaction $(P<0.001)$. (b) Plasma insulin: there was a trend in favour of a diet $\times$ time interaction $(P=0.091)$. (c) Plasma TAG: there were significant main effects of diet $(P<0.001)$ and a diet $\times$ time interaction $(P<0.001)$. Significant differences found between: iso $v$. total ER $(P=0.001)$ and partial ER $(P=0.005)$. (d) Plasma NEFA: there were significant main effects of diet $(P=0.008)$ and a diet $\times$ time interaction $(P<0.001)$. Differences found between total ER $v$. iso $(P=0.039)$ and partial ER $(P=0.083$; non-significant trend). Comparisons (using plasma concentration) made using repeated-measures ANOVA (Sidak correction applied). Values are mean change from baseline $(n 10)$, with their standard errors. 
(a)

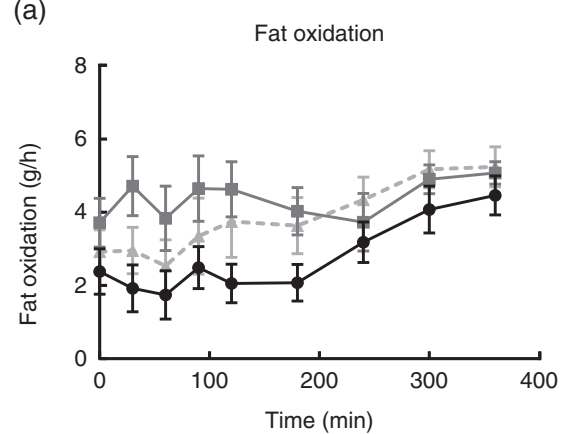

(b)

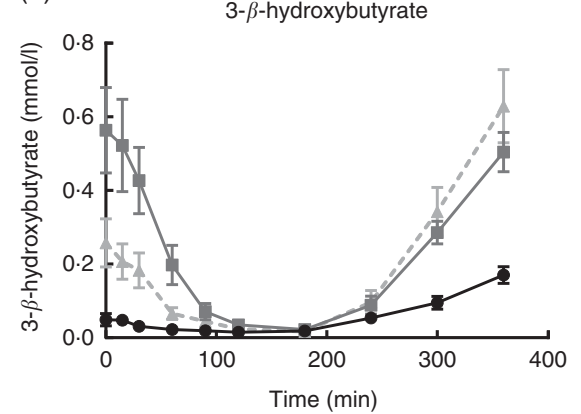

(c)

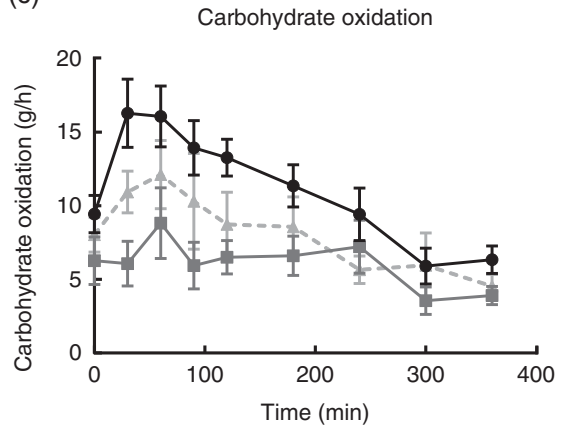

Fig. 3. Postprandial (6-h) substrate oxidation responses to liquid test meal ( $2510 \mathrm{~kJ}$ ( $600 \mathrm{kcal}), 74 \mathrm{~g}$ carbohydrate, $24 \mathrm{~g}$ protein, $23 \mathrm{~g}$ fat) following $1 \mathrm{~d}$ of total $100 \%$ energy restriction (ER) (- - ) and partial $75 \% \mathrm{ER}\left(-\mathrm{A}_{-}^{-}\right) \mathrm{v}$. an isoenergetic (iso) control diet (- - ). (a) Fat oxidation (indirect calorimetry): there was a trend in favour of a main effect of diet $(P=0.080)$. No significant pairwise differences found between iso (17 (SEM 2) g), total ER (26 (SEM 4) g; $P=0.184)$ or partial ER (24 (SEM 4) g; $P=0.233$ ) interventions. (b) Plasma 3- $\beta$-hydroxybutyrate: there were significant main effects of diet $(P<0.001)$ and a diet $\times$ interaction $(P<0.001)$ for postprandial 3- $\beta$-hydroxybutyrate responses. Significant differences found between iso $v$. total ER $(P=0.001)$ and partial ER $(P=0.007)$. (c) Carbohydrate oxidation (indirect calorimetry): there were significant main effects of diet $(P=0.023$ ). Trends found between iso (59 (SEM 5) g) $v$. total ER (32 (SEM 6) g; $P=0.051)$ but not partial ER (43 (SEM 7) g; $P=0.174)$. Comparisons made using repeated-measures ANOVA (Sidak correction applied). Values are means $(n 8$, indirect calorimetry data; $n$ 10, 3- $\beta$-hydroxybutyrate), with their standard errors.

v. 1 (SEM 4) $\mathrm{mmol} / \mathrm{min}$ per litre; $P=0.015)$ and partial ER $(-33$ (SEM 16) $\mathrm{mmol} / \mathrm{min}$ per litre; $P=0 \cdot 015$ ). dAUC did not differ significantly between partial ER and iso interventions. No statistically significant differences in resting energy expenditure (REE) or MIT were detected across the dietary interventions (data not presented).

\section{Discussion}

Our data demonstrate a number of distinct alterations to postprandial substrate metabolism, assessed on the morning after $1 \mathrm{~d}$ of total $(100 \%)$ and partial $(75 \%) \mathrm{ER}$, which were evident following both levels of ER. Very few studies have quantified the metabolic and physiological changes that occur in response to substantial ER. These acute disturbances in fuel management could lead to changes in so-called metabolic flexibility, a marker of metabolic health. As a result, regimens incorporating repeated bouts of substantial ER could lead to altered cardiometabolic risk, independent of weight change.

\section{Postprandial glucose metabolism}

Our finding that oral glucose tolerance was impaired after $1 \mathrm{~d}$ (36h) of total ER is in accordance with the existing research on prolonged fasting, which up to now has either involved lean participants or more prolonged $(\geq 48 \mathrm{~h})$ fasting intervals ${ }^{(13,14,16)}$.
We additionally found a significant delay in the postprandial glucose curve, which, in the context of early type 2 diabetes mellitus, is associated with impairments in $\beta$-cell function and insulin secretion ${ }^{(25)}$. Indeed, postprandial insulin profiles following both ER interventions appeared flattened and prolonged; however, without measuring C-peptide levels, we were unable to assess the individual effects on insulin secretion $v$. hepatic clearance.

In the present study, the prolonged 36-h fast was accompanied by a significant elevation in circulating plasma NEFA, which has been implicated as a key driver behind our observations; directly, peripheral tissue accumulation of NEFA and/or its derivatives (ceremides, diacylglycerol) are linked to disruptions in peripheral insulin signalling and/or glucose transport ${ }^{(26,27)}$, as well as the acute insulin response ${ }^{(16)}$; indirectly, the associated rise in fat oxidation and ketosis are also capable of impairing peripheral glucose disposal ${ }^{(14,28)}$. Previously, Salgin et $a l^{(16)}$. showed that treatment with an antilipolytic agent (acipimox) could only partially reverse fast-induced effects on insulin secretion and peripheral insulin sensitivity, highlighting the role of other contributory mediators such as circulating counter-regulatory hormones, which are also elevated after prolonged fasts ${ }^{(16,29)}$.

In an acute sense, our findings can be viewed as an adaptive physiological response to short-term starvation, one which allows for a shift in postprandial nutrient partitioning in favour of glucose conservation and glycogen repletion ${ }^{(11)}$. Whether there may be tachyphylaxis of these effects or metabolic adaption with repeated 
spells of ER re-feeding is unknown. The premise of IER is that there is long-term adaptation to the repeated elevations in NEFA, leading to the up-regulation of mitochondrial FAO in skeletal muscle. This has the potential to improve insulin sensitivity by reducing the accumulation of lipid intermediaries over time. In lean or overweight humans, one short-term study using 3 weeks of alternate-day total fasting demonstrated trends for up-regulation of the mitochondrial fatty acid transporter CPT-1 within skeletal muscle, but conversely a decline in mitochondrial DNA copy number (a marker of mitochondrial function) ${ }^{(5)}$. Also observed by this study was an impairment in glucose tolerance among women; however, the uneven pre- and post-intervention fasting intervals used in this study (12 v. 36h respectively) make it difficult to ascribe this as a true chronic treatment effect. Animal models using a similar alternate-day fasting protocol also report increases in insulin receptor nitration in skeletal muscle during long-term IER, which was accompanied by a reduction in glucose tolerance ${ }^{(30)}$.

The partial (75\%) ER diet was designed to place participants in a severe negative energy balance while allowing some food intake. This consequently blunted the progressive rise in plasma NEFA seen with prolonged fasting intervals. Accordingly, observed alterations in postprandial glucose tolerance and nutrient oxidation effectively displayed a dose-response across the two levels of substantial (75-100\%) ER, with partial ER attenuating the impairments to glycaemic control seen following a day of total ER. Ultimately, in humans, the significance of short-term increases in NEFA and impaired glucose tolerance with fasting is unknown; whether repeated spells of total ER present too great a metabolic perturbation; and if any potential long-term adverse health implications of this could be avoided through partial ER warrant further investigation.

\section{Postprandial TAG metabolism}

We observed favourable alterations to TAG metabolism following both degrees of substantial ER, which has not been shown previously in overweight/obese participants. By combining lines of evidence from our study and others, we propose a number of major contributory mechanisms: first, the liver is highly sensitive to changes in energy status. Profound negative energy deficits have been shown to partition fatty acids derived from circulatory TAG, NEFA and intra-hepatocellular (IHCL) stores towards $\beta$-oxidation or ketogenesis $^{(31)}$. Indeed, fasting levels of $3-\mathrm{OHB}$ were elevated after both ER interventions. Fatty acid availability for TAG re-esterification, storage as IHCL and VLDL-TAG secretion is thus limited, as evidenced by studies of regional glycerol turnover during prolonged fasting, which have shown rates of hepatic non-oxidative NEFA disposal to be comparatively lower than that of peripheral tissues ${ }^{(32)}$. Accordingly, we observed acute reductions in fasting TAG, which predominantly reflects an ER-induced decrease in fasting VLDL-TAG secretion ${ }^{(33)}$. A major regulator of ketogenesis in humans is the supply of fatty acids (34). Our metabolic (NEFA and 3-OHB) and substrate oxidation data closely parallel each other and are suggestive of a shift in postprandial partitioning of fatty acids towards hepatic ketone body synthesis (away from VLDL-TAG secretion) and FAO (facilitating glycogen repletion). In the postprandial state, chylomicron-TAG (derived from dietary sources) are preferentially cleared, and thus VLDL-TAG make up approximately $90 \%$ of TAG in the postprandial state ${ }^{(35)}$. A reduction in fasting and postprandial VLDL-TAG would be expected to reduce the overall magnitude of the postprandial response via reduced competition between endogenously and exogenously derived lipids for clearance via a common, saturable lipolytic pathway ${ }^{(36,37)}$.

\section{Energy intake compensation and energy expenditure}

To contextualise to a situation of weight management, our secondary aims were to study the acute effects of different degrees of ER on acute compensation in energy intake and components of energy expenditure. Our food intake data suggest that the participants were still in negative energy balance after $2 \mathrm{~d}$ of ad libitum intake, which is particularly interesting, given that individuals following IER often undergo repeated ER/feed cycles over the course of a week. We are the first to show that both total and partial ER can produce comparable short-term energy intake deficits of approximately $30 \%$ in overweight/obese participants, with no additional deficit achieved by fasting completely. Anecdotally, it is likely that protocols that allow a small amount of food intake are likely to be better tolerated over time, although this has not been directly assessed. Our data are in line with other studies that have similarly demonstrated an apparent lack of tight physiological control in day-to-day energy balance following substantial ER within lean participants with no/mild eating restraint ${ }^{(38,39)}$ and in overweight participants during chronic $\operatorname{IER}^{(3,4)}$.

On the other side of the energy balance equation, we found no compensatory declines in REE or MIT. To date, acute data on energy expenditure have been equivocal ${ }^{(15,40-42)}$, with fastinduced increases in mitochondrial uncoupling ${ }^{(43)}$, sympathetic nervous system activation and catecholamine levels ${ }^{(42)}$ thought to contribute towards the paradoxical increases in REE found by some studies ${ }^{(15,41,42)}$. Although our assessments of energy expenditure are limited to REE and MIT responses to a single meal, others have reported $\sim 6 \%$ reductions in 24-h energy expenditure after prolonged fasts using wholebody calorimeters ${ }^{(44,45)}$. However, any small changes to energy expenditure are unlikely to offset the larger changes in energy intake.

\section{Strengths and limitations}

A particular strength of this study is the novel use of a withinsubject study design to compare individual responses to varying levels of substantial ER, while allowing participants to act as their own control. From a statistical standpoint, although this study design somewhat mitigates the small heterogeneous group used by removing the inter-individual variation element, the study (originally powered on postprandial TAG) is likely to be underpowered with respect to our indirect calorimetry data. This is exemplified by the loss of statistical significance with our substrate oxidation time-course data following multiple post hoc comparisons. Owing to the sample size and relatively small proportion of women, it was not possible to fully explore sex differences. Sex dimorphism in fasting responses have previously been shown, with women tending to display greater NEFA mobilisation owing to differences in adiposity ${ }^{(46)}$. Finally, our 
postprandial assessments were limited to measuring changes in absolute substrate concentrations after a single meal, which represents the balance but not the rate (or source) of substrate appearance or clearance.

\section{Summary and future research directions}

Put together, our data demonstrate the ability of substantial (75-100\%) ER to acutely alter glucose-lipid metabolism, as well as incomplete short-term energy intake compensation amongst overweight/obese participants. Partial ER (as compared with total ER) reduced the fast-associated decline in oral glucose tolerance, whilst producing a comparable 3-d energy-intake deficit and improvement in postprandial TAG. The experimental partial ER diet was low in carbohydrate (37\% of total energy); perhaps a greater carbohydrate supply on ER days, through further attenuation of this starvation response, may have ameliorated the negative short-term effects on glycaemia. It should be noted that our findings represent the acute postprandial changes after a single test meal in a group of healthy overweight/obese individuals. Whether there is tachyphylaxis or metabolic adaptation to the repeated spells of ER re-feeding, and if any potential harm of the repeated fast-associated elevations in NEFA and impaired glucose tolerance can be mitigated through partial ER, are unknown. Further investigations are required to establish how metabolism adapts over time to the repeated perturbations experienced during IER, as well as the implications of our findings (both positive and negative) for long-term health.

\section{Acknowledgements}

The authors thank the staff at the Surrey Clinical Research Centre and BSc/MSc project students for their assistance during the trial, as well as the participants who completed the trial.

K. L. J. is Head of Nutrition and Research at Lighterlife ${ }^{\circledR}$. This work was supported by Lighterlife ${ }^{\circledR}$ who funded the running costs of the trial and provided the FoodPacks used during the partial ER intervention.

The authors' contributions are as follows: R. A. was involved in the study conception, design, running of the study, laboratory work, statistical analysis and manuscript preparation; K. L. J. assisted with manuscript preparation; A. L. C. was involved in the study conception, design and manuscript preparation; and M. D. R. was involved in the study conception, design, statistical analysis and manuscript preparation.

\section{References}

1. Antoni R, Johnston KL, Collins AL, et al. (2014) The effects of intermittent energy restriction on indices of cardiometabolic health. Res Endocrinol 2014, Article ID 459119.

2. Klempel MC, Kroeger CM, Bhutani S, et al. (2012) Intermittent fasting combined with calorie restriction is effective for weight loss and cardio-protection in obese women. Nutr J 11, 98.

3. Harvie MN, Pegington M, Mattson MP, et al. (2011) The effects of intermittent or continuous energy restriction on weight loss and metabolic disease risk markers: a randomised trial in young overweight women. Int J Obes 35, 714-727.
4. Harvie M, Wright C, Pegington M, et al. (2013) The effect of intermittent energy and carbohydrate restriction $\mathrm{v}$. daily energy restriction on weight loss and metabolic disease risk markers in overweight women. Br J Nutr 110, 1534-1547.

5. Heilbronn LK, Civitarese AE, Bogacka I, et al. (2005) Glucose tolerance and skeletal muscle gene expression in response to alternate day fasting. Obes Res 13, 574-581.

6. Bhutani S, Klempel MC, Kroeger CM, et al. (2013) Alternate day fasting and endurance exercise combine to reduce body weight and favorably alter plasma lipids in obese humans. Obesity (Silver Spring) 21, 1370-1379.

7. Hyson D, Rutledge JC \& Berglund MD (2003) Postprandial lipemia and cardiovascular disease. Cur Atheroscler Rep $\mathbf{5}$, 437-444.

8. Node K \& Inaeu T (2009) Postprandial hyperglycemia as an etiological factor in vascular failure. Cardiovasc Diabetol $\mathbf{8}, 23$.

9. Lara-Castro C, Newcomer B \& Rowell J (2008) Effects of shortterm very low calorie diet on intramyocellular lipid and insulin sensitivity in non-diabetics and type 2 diabetic patients. Metabolism 57, 1-8.

10. Lim EL, Hollingsworth KG, Aribisala BS, et al. (2011) Reversal of type 2 diabetes: normalisation of beta cell function in association with decreased pancreas and liver triacylglycerol. Diabetologia 54, 2506-2514.

11. Soeters MR, Soeters PB, Schooneman MG, et al. (2012) Adaptive reciprocity of lipid and glucose metabolism in human short-term starvation. Am J Physiol Endocrinol Metab 303, E1397-E1407.

12. Anderson JW \& Herman RH (1972) Effect of fasting, caloric restriction, and refeeding on glucose tolerance of normal men. Am J Clin Nutr 25, 41-52.

13. Fery F, d'Attellis NP \& Balasse EO (1990) Mechanisms of starvation diabetes: a study with double tracer and indirect calorimetry. Am J Physiol 259, E770-E777.

14. Horton TJ \& Hill JO (2001) Prolonged fasting significantly changes nutrient oxidation and glucose tolerance after a normal mixed meal. I Appl Physiol 90, 155-163.

15. Bergman BC, Cornier MA, Horton TJ, et al. (2007) Effects of fasting on insulin action and glucose kinetics in lean and obese men and women. Am J Physiol Endocrinol Metab 293, E1103-E1111.

16. Salgin B, Marcovecchio ML, Humphreys SM, et al. (2009) Effects of prolonged fasting and sustained lipolysis on insulin secretion and insulin sensitivity in normal subjects. Am J Physiol Endocrinol Metab 296, E454-E461.

17. van Strien T, Frijters JER, Bergers GPA, et al. (1986) The Dutch Eating Behavior Questionnaire (DEBQ) for assessment of restrained, emotional, and external eating behavior. Int J Eat Disord 5, 295-315.

18. Henry CJ (2005) Basal metabolic rate studies in humans: measurement and development of new equations. Public Health Nutr 8, 1133-1152.

19. Scientific Advisory Committee on Nutrition (SACN) Energy Requirements Working Group (2011) Dietary reference values for energy. https://www.gov.uk/government/uploads/system/ uploads/attachment_data/file/339317/SACN_Dietary_Reference_ Values_for_Energy.pdf (accessed July 2015).

20. Nicholson MJ, Holton J, Bradley AP, et al. (1996) The performance of a variable-flow indirect calorimeter. Physiol Meas 17, 43-55.

21. Compher C, Frankenfield D, Keim N, et al. (2006) Best practice methods to apply to measurement of resting metabolic rate in adults: a systematic review. J Acad Nutr Diet 106, 881-903.

22. Weir JB (1949) New methods for calculating metabolic rate with special reference to protein metabolism. J Physiol 109, 1-9. 
23. Frayn KN (1983) Calculation of substrate oxidation rates in vivo from gaseous exchange. J Appl Physiol Respir Environ Exerc Physiol 55, 628-634.

24. Brouns F, Bjorck I, Frayn KN, et al. (2005) Glycaemic index methodology. Nutr Res Rev 18, 145-171.

25. Kramer CK, Vuksan V, Choi H, et al. (2014) Emerging parameters of the insulin and glucose response on the oral glucose tolerance test: reproducibility and implications for glucose homeostasis in individuals with and without diabetes. Diabetes Res Clin Prac 105, 88-95.

26. Roden M, Price TB, Perseghin G, et al. (1996) Mechanism of free fatty acid-induced insulin resistance in humans. $J$ Clin Inves 97, 2859-2865.

27. Holland WL, Knotts TA, Chavez JA, et al. (2007) Lipid mediators of insulin resistance. Nutr Rev 65, S39-S46.

28. Webber J, Simpson E, Parkin H, et al. (1994) Metabolic effects of acute hyperketonaemia in man before and during an hyperinsulinaemic euglycaemic clamp. Clin Sci (Lond) 86, 677-687.

29. McMahon M, Gerich J \& Rizza R (1988) Effects of glucocorticoids on carbohydrate metabolism. Diabetes Metab Rev 4, $17-30$.

30. Cerqueira FM, da Cunha FM, Caldeira da Silva CC, et al. (2011) Long-term intermittent feeding, but not caloric restriction, leads to redox imbalance, insulin receptor nitration, and glucose intolerance. Free Radic Biol Med 51, 1454-1460.

31. Plaisance EP \& Fisher G (2014) Exercise and dietary-mediated reductions in postprandial lipemia. J Nutr Metab 2014, 902065.

32. Jensen MD, Ekberg K \& Landau BR (2001) Lipid metabolism during fasting. Am J Physiol Endocrinol Metab 281, E789-E793.

33. Bellou E, Maraki M, Magkos F, et al. (2013) Effect of acute negative and positive energy balance on basal very-low density lipoprotein triglyceride metabolism in women. PLOS ONE 8, e60251.

34. Frayn K, Humphreys S \& Coppack S (1996) Net carbon flux across subcutaneous adipose tissue after a standard meal in normal-weight and insulin resistant obese subjects. Int J Obes Relat Metab Disord 20, 795-800.
35. Karpe F, Hellénius M \& Hamsten A (1999) Differences in postprandial concentrations of very-low-density lipoprotein and chylomicron remnants between normotriglyceridemic and hypertriglyceridemic men with and without coronary heart disease. Metabolism 48, 301-307.

36. O'Meara N, Lewis G, Cabana V, et al. (1992) Role of basal triglyceride and high density lipoprotein in determination of postprandial lipid and lipoprotein responses. J Clin Endocrinol Metab 75, 465-471.

37. Griffin BA (2013) Lipid metabolism. Surgery (Oxford) 31, 267-272.

38. Johnstone AM, Faber P, Gibney ER, et al. (2002) Effect of an acute fast on energy compensation and feeding behaviour in lean men and women. Int J Obes Relat Metab Disord 26, 1623-1628.

39. Levitsky DA \& DeRosimo L (2010) One day of food restriction does not result in an increase in subsequent daily food intake in humans. Physiol Behav 99, 495-499.

40. Nair K, Woolf P, Welle S, et al. (1987) Leucine, glucose, and energy metabolism after 3 days of fasting in healthy human subjects. Am J Clin Nutr 46, 557-562.

41. Mansell P \& Macdonald I (1990) The effect of starvation on insulin-induced glucose disposal and thermogenesis in humans. Metabolism 39, 502-510.

42. Webber J \& Macdonald I (1994) The cardiovascular, metabolic and hormonal changes accompanying acute starvation in men and women. BrJ Nutr 71, 437-447.

43. Hoeks J, van Herpen NA, Mensink M, et al. (2010) Prolonged fasting identifies skeletal muscle mitochondrial dysfunction as consequence rather than cause of human insulin resistance. Diabetes 59, 2117-2125.

44. Dauncey MJ (1980) Metabolic effects of altering the 24-h energy-intake in man, using direct and indirect calorimetry. Br J Nutr 43, 257-269.

45. de Boer JO, Vanes AJH, Roovers LCA, et al. (1986) Adaptation of energy-metabolism of overweight women to low-energy intake studied with whole-body calorimeters. Am J Clin Nutr 44, 585-595.

46. Mittendorfer B, Horowitz J \& Klein S (2001) Gender differences in lipid and glucose kinetics during short-term fasting. Am J Physiol Endocrinol Metab 281, E1333-E1339. 